\title{
Laboratorio virtual de matemáticas como estrategia didáctica para fomentar el pensamiento lógico ${ }^{1}$
}

\author{
Sandra Liliana Torres Taborda ${ }^{2}$ \& Efraín José Martínez Meneses ${ }^{3}$ \\ Corporación Universitaria de Sabaneta (UniSabaneta), Colombia
}

Recibido, mayo 19 de 2015

Concepto evaluación, agosto 03 de 2015

Aceptado, octubre 15 de 2015
Referencia: Torres Taborda, S.; Martínez Meneses, E. (2015). "Laboratorio virtual de matemáticas como estrategia didáctica para fomentar el pensamiento lógico". Revista Academia y Virtualidad, 8, (2), 73-84

\section{Resumen}

Las instituciones de Educación Superior se enfrentan constantemente al bajo rendimiento alcanzado por sus estudiantes en el área de matemáticas, situación que se deriva de factores como la cantidad de horas dedicadas según el currículo, número de docentes y de personas por curso, al igual que ausentismo escolar o apatía por el área de formación. El laboratorio virtual de matemáticas es una herramienta versátil que busca complementar los procesos de inclusión educativa, permitiendo que los estudiantes en su heterogeneidad de conocimientos y habilidades matemáticas puedan lograr al final de su proceso académico un nivel semejante de competencias matemáticas.

A través de la solución de situaciones problémicas apoyadas en las herramientas de laboratorio (objetos virtuales de aprendizaje-OVA), un grupo focal lleva a cabo la prueba durante cuatro meses, período durante el cual se le ofrece posibilidades de encuentros sincrónicos y diversas herramientas didácticas para mejorar su nivel de autoconfianza y la comprensión matemática de las situaciones que enfrenta cotidianamente (matematización del pensamiento).

Palabras clave: análisis matemático, aprendizaje de adultos, educación a distancia, método de aprendizaje, razonamiento.

1. Artículo de reflexión en el marco de la investigación que lleva el mismo nombre y que se realizó como trabajo de grado de la maestría en E-Learning de la Universidad Autónoma de Bucaramanga en convenio con la Universitat Oberta de Catalunya para el Grupo de Investigación EDUTEC.

2. Negociadora Internacional, especialista en Alta Gerencia y candidata a Magíster en E-Learning. Formada como tutora virtual por la Organización de Estados Americanos OEA en convenio con el Ministerio de Educación Nacional de Colombia. Con experiencia docente y administrativa en Educación Superior. Investigadora de los grupos POLEMOS y GIDEMP de la Corporación Universitaria de Sabaneta, UniSabaneta. Correo: storres139@unab.edu.co

3. Ingeniero, Universidad de Antioquia, candidato a Magíster en E-Learning. Siete años de experiencia Educación Superior. Investigador de los grupos POLEMOS y GIDEMP de la Corporación Universitaria de Sabaneta, UniSabaneta; grupo ICONO de la institución Universitaria Tecnológico Pascual Bravo y gestor TIC por el Ministerio de las TIC de Colombia. Correo: emartinez102@unab.edu.co 


\section{A virtual math lab as a teaching strategy to promote logical thinking}

\section{Abstract}

Higher education entities are continually faced to low performance rates by their students in mathematics, resulting from factors such as number of hours according to the curriculum; number of teachers and students per course; truancy; or apathy on subject. A virtual math lab is a versatile tool to supplement processes of educational inclusion, allowing students depending on their math knowledge and skills may achieve at the end of the academic process a similar level of math competencies.

By solving problem situations supported by lab tools (virtual learning objects-VLO), a focus group performs a test for four months, a period during which synchronous meetings and several educational tools are given to improve selfconfidence level and mathematical understanding related to daily situations faced (mental mathematization.)

Keywords: mathematical analysis, adult learning, distance education, learning methods, reasoning.

\section{Laboratório virtual de matemáticas como estratégia didática para o fomento do pensamento lógico}

\section{Resumo}

As instituições de Educação Superior enfrentam-se constantemente ao baixo rendimento alcançado pelos seus estudantes na área de matemáticas, situação derivada de fatores como a quantidade de horas dedicadas segundo o curriculum, número de docentes e de pessoas por curso, assim como o absentismo escolar ou apatia pela área de formação. O laboratório virtual de matemáticas é uma ferramenta versátil que procura complementar os processos de inclusão educativa, permitindo aos estudantes, na sua heterogeneidade de conhecimentos e habilidades matemáticas, atingir ao final do seu processo académico um nível semelhante de competências matemáticas.

Através da solução de situações problemas apoiadas nas ferramentas de laboratório (objetos virtuais de aprendizagemOVA), um grupo focal operacionaliza a prova durante quatro meses, período durante o qual thes são oferecidas possibilidades de encontros sincrônicos e diversas ferramentas didáticas para melhorar seu nível de autoconfiança e a compreensão matemática das situações que enfrenta quotidianamente (matematização do pensamento).

Palavras chave: análise matemática, aprendizagem de adultos, educação a distância, método de aprendizagem, razoamento. 


\section{Introducción}

La búsqueda de la inclusión educativa ha permitido el acceso de personas que debido a sus características socioeconómicas no podían tener una carrera universitaria en su proyecto de vida; así, surgen y se adaptan instituciones a las condiciones horarias, geográficas $\mathrm{y}$ al poder adquisitivo de un sector importante de la sociedad. Desde algunos puntos de vista es un avance positivo en búsqueda de la equidad, pero esta situación trae consigo escenarios, tales como personas con ceses académicos prolongados y personas que al no tener entre sus objetivos el ingreso a la Educación Superior tuvieron un desempeño escolar descuidado, y que en su mayoría poseen poca resistencia al error (Bastán \& Elguero, 2005). Los docentes se enfrentan entonces a individuos con muchas habilidades y conocimientos matemáticos, a personas con falencias considerables y a otras sin manejo de herramientas y conceptos, todos dentro de una misma aula de clase.

La preocupación de las instituciones de Educación Superior por el bajo rendimiento en matemáticas es permanente, pero se ha focalizado en el rol del estudiante. Las estrategias de solución son repetitivas y fundamentadas en la cantidad de horas dedicadas según el currículo, o en el número de docentes y de personas por curso. El temor y la frustración derivados del ausentismo escolar suele generar en los educandos apatía por el aprendizaje, haciéndose necesario el apoyo en diferentes estrategias pedagógicas que propendan por el desarrollo de habilidades y conocimientos concretos que permitan contrarrestar estas deficiencias. Por fortuna, la mediación de las TIC en la educación ofrece una interesante gama de posibilidades, entre ellas, los materiales educativos multimedia (MEM). No obstante, ¿cómo pueden los MEM facilitar la comprensión de las matemáticas? La diversidad de canales comunicativos que ofrece multimedia promueve la asimilación del conocimiento, acceso a recursos en el momento de preferencia del estudiante y cuantas veces lo considere necesario sin temor a ser juzgado, además de generar confianza y promover el autoaprendizaje al permitir explorar, seleccionar, analizar y hasta jugar con la información suministrada.
El propósito del laboratorio virtual de matemáticas es reforzar el proceso educativo y facilitar el desarrollo de competencias matemáticas contrarrestandolas condiciones de heterogeneidad académica de los estudiantes. Para el desarrollo de nuestro Sistema de Gestión de Aprendizaje (LMS), nos apropiamos del concepto que la pedagogía como disciplina debe adaptarse a las características del estudiante, no a las del profesor y consideramos que para propiciar la nivelación académica, es conveniente el uso de recursos creados como soporte para favorecer la experiencia educativa; de manera que decidimos hacer nuestros propios objetos virtuales de aprendizaje (OVA) apoyados en la tecnología multimedia.

El laboratorio propone diferentes niveles de complejidad en los procesos de aprendizaje, para que cada estudiante identifique en qué estado se encuentran sus habilidades y conocimientos, busque constructivamente un punto óptimo que lo homogenice con el grupo y permitirle al docente avanzar en las asignaturas sin dejar personas rezagadas, propensas a la deserción temprana (Castaño, Gómez, Gallón \& Vásquez; 2008) o sometidas al tedio por la repetición de fundamentos teóricos ya dominados.

Así, se crea un portal web como LMS en html 5, de tal manera que se adapta a los diversos dispositivos móviles y computadores tradicionales sin perder sus cualidades y posibilidades de accesibilidad a los cursos y otros enlaces, diseñamos OVA como soporte teórico usando tabletas de dibujo de referencias Imousepen 608x y G-Pen F509 marca Genius, la herramienta Hot Potatoes, programas como Paint.net, Camtasia y recursos de animación gratuita de Powtoon.com; todo esto apoyado en el método de aprendizaje basado en problemas con metas sencillas y significativas para los aprendices.

El trabajo entonces involucra la participación de un grupo focal de seis estudiantes universitarios de primer semestre, con ceses académicos prolongados y con responsabilidades laborales de tiempo completo; pertenecientes a la Corporación Universitaria de Sabaneta J. Emilio Valderrama-UniSabaneta (Antioquia) para impactarlos no sólo desde el aspecto de las denominadas competencias matemáticas, sino también como proceso 
de sensibilización y ruptura de paradigmas con respecto a la imposibilidad de aprender matemáticas en ambientes virtuales de aprendizaje. No obstante, el laboratorio virtual tuvo durante el semestre II de 2014 un total de 73 estudiantes matriculados quienes reafirmaron el impacto positivo en su proceso académico y, como consecuencia, el laboratorio fue reconocido por la oficina de Bienestar Universitario de la institución como una herramienta del programa "cero deserción" y fue presentado como tal ante la visita de pares académicos del Ministerio de Educación Nacional en el mes de noviembre para la renovación de registro calificado de algunos programas.

Además de potenciar la capacidad de resolver problemas $\mathrm{y}$ favorecer los procesos cognitivos deductivos; las herramientas multimedia y recursos web ayudan para que el entorno sea suficiente, claro, intuitivo, amigable y permita eventos sincrónicos de apoyo y discusión (Granados, Ureña \& Gamba. 2009). El medio electrónico le permite al estudiante acceder a éste de manera individual y realizar sus actividades, reconocer sus limitaciones y superar sus falencias sin las presiones que presupone estar en un aula de clase, y al docente poder tener comunicación exclusiva y singular con su estudiante.

\section{Estado del arte}

La digitalización en que vivimos necesariamente innova el proceso formativo para docentes y discentes, abre nuevas oportunidades y métodos para enseñar y para aprender, exige una planeación diferente y consciente de la interactividad permanente en la que está inmerso el estudiante. Así,

[...] El conocimiento, desde esta perspectiva, está en constante transformación y los miembros de cada generación se apropian de él, en cada sociedad, con el propósito de darle solución a nuevos problemas. El conocimiento no es invariable y estático, es parte integral y dinámica de la vida misma, de las indagaciones que los miembros de una sociedad hacen acerca de sus condiciones, sus preocupaciones y sus propósitos (Chaves \& Peña, 2006: 27).
El material que usualmente se emplea en el desarrollo cotidiano de las clases presenciales y que se compone de módulos temáticos, ejercicios de aplicación y momentos evaluativos puede igualmente ponerse a disposición de otras comunidades académicas o de un público abierto a través de material educativo multimedia (MEM) empleado como apoyo del E-Learning.

El E-Learning toma sustancial importancia al convertirse en un elemento de uso cada vez más frecuente en la educación, bien sea como apoyo para dar cumplimiento a las actividades de trabajo independiente que debe desarrollar el estudiante según la concepción de créditos académicos o bien, como columna vertebral del proceso formativo bajo el modelo de educación a distancia. Los materiales educativos multimedia son un excelente apoyo para la implementación de la educación.

Para enfrentar un proyecto para la enseñanza de las matemáticas por medio de material multimedia se debe transitar por tres rutas que deben llegar al final a una misma meta y todas tres deben ser recorridas para no cercenar el producto final. Estos tres factores generales para tener en cuenta son:

\section{Políticas estatales y privadas}

Las políticas en relación con las TIC en la educación en Latinoamérica y el Caribe, vienen con tres promesas claras: la alfabetización digital, disminuir la brecha tecnológica e implementar nuevas estrategias para la enseñanza (Claro, 2010). Esto nos lleva a plantear necesidades urgentes para la Educación Superior y para la didáctica moderna. En nuestro caso particular, generar material multimedia para la enseñanza de las matemáticas no resulta una innovación propiamente dicha, sino una adaptación y una actualización de recursos.

\section{Tecnología}

La aplicación de las nuevas tecnologías a la enseñanza se ha convertido en una preocupación de los Estados desde hace más de 15 años, tal como lo plantea el informe de la $\mathrm{Cepal}^{4}$ sobre el impacto de las TIC en el aprendizaje 
de los estudiantes, que continúa reforzando el cambio de paradigma del tradicional método magistral al aula interactiva donde el emisor y el receptor se confunden por la tecnología. Si bien no hay que caer en la sobrevaloración de las nuevas posibilidades multimediales porque como lo demostraron las pruebas PISA $^{5}$, el acceso por sí solo a las nuevas tecnologías no asegura mejores resultados en el aprendizaje de las matemáticas, sigue siendo la manera en que éstas se utilizan las que brindan un impulso positivo a los nuevos elementos del posmodernismo educativo.

Asimismo, se habla de las TAC (tecnologías del aprendizaje y el conocimiento), según Steegmann, Pérez y Huertas (2004), como una rama dentro del gran campo de la educación y la didáctica que busca brindar complementos y diversidad a la enseñanza de las matemáticas apoyándose en el uso del internet, los asistentes matemáticos y todas las posibilidades multimediales de video, hipertexto, sonido, animación, pizarras electrónicas, etc. Todo ello configurando un espectro del E-Learning especializado en herramientas para buscar impactar positivamente la aprehensión del conocimiento y el razonamiento matemático.

\section{Psicopedagogía}

La creación de un buen objeto no sólo depende del diseñador y las herramientas tecnológicas, sino también de todas las implicaciones cognitivas que trae consigo el evento de enseñanza-aprendizaje mediado o apoyado por la tecnología, tal como lo plantea The Cambridge handbook of multimedia learning (Mayer, 2005), donde la memoria y la manera de asimilar los diferentes escenarios multimediales poseen en sí mismos sus complejidades y fundamentan sicológica y pedagógicamente el diseño, la construcción y la puesta en marcha de un objeto o material multimedia para la enseñanza de la matemática en la universidad.

Cuando se pretende construir y transmitir conocimiento, existe inicialmente la necesidad de comunicarnos; más aún, que esta comunicación sea efectiva, de tal forma que el receptor se apropie del mensaje en cuestión. Resolver el porqué y el cómo es un ejercicio semiótico indispensable en el abordaje de cualquier elaboración de material multimedia (Chavarría \& Carvajal, 2008).

En el caso del pensamiento lógico, del pensamiento matemático, se debe tener especial cuidado porque el temor generalizado hacia las mismas y el paradigma de la presencialidad que las vuelve dependiente de la explicación personalizada, obligan a mantener un lenguaje persuasivo y amigable sin abandonar en ningún momento el lenguaje especializado y universal de las matemáticas. El reto consiste no sólo en el uso de estas herramientas sino en la forma como son utilizadas para potenciar el aprendizaje en los estudiantes; específicamente, la enseñanza de las matemáticas.

La implementación de la multimedia es benéfica gracias a que ésta se destaca por su versatilidad y por el amplio espectro de aplicabilidad gracias a que puede integrar elementos que propician la interactividad y por ende, fomentan la interconexión. Mayer (2005) afirma que un mensaje instruccional multimedia es una comunicación con palabras e imágenes destinadas a fomentar el aprendizaje.

Los materiales educativos multimedia no deben ser complejos a fin de propiciar su comprensión. García (citado por García y Benítez, 2011: 2) considera que es conveniente tomar en cuenta que para integrar alguna tecnología digital, los estudiantes deberán desarrollar competencias relacionadas con el manejo de las TIC, para que su integración contribuya al logro de los aprendizajes propuestos.

El desafío central para los diseñadores de instrucción multimedia es potenciar el desarrollo cognitivo del alumno (Mayer \& Moreno, 2003), pues la influencia del E-Learning se centra en el estudiante y no en el docente,

4. Cepal (Comisión Económica para América Latina y el Caribe). Impacto de las TIC en los aprendizajes de los estudiantes, estado del arte, documentos de proyectos.

5. PISA (Program for International Student Assessment) y la evaluación de las matemáticas, Revista de educación. 
haciendo necesario que las tecnologías empleadas posean un diseño instruccional acorde con las características del público objetivo.

Debido a la revolución de las tecnologías de la información basadas en internet, resulta casi obvio la utilización del hipertexto como herramienta fundamental en un laboratorio de matemáticas basado en multimedios, sin que sea un enfrentamiento contra la didáctica tradicional, simplemente se trata de una adaptación de los conceptos y las estrategias (Cangiani \& Zabert, 2008). La creación de software especializado como el caso de Mathematicas (Malaina \& Alboniga, 2006) y Phi (Moya \& González, 2006), es una opción como laboratorio pues ofrecen interactividad y pertinencia temática enfocados en la resolución de problemas, pero continúa limitando las posibilidades y las herramientas. Por ello,

[...] El uso de los laboratorios virtuales permite generar nuevos espacios pedagógicos interactivos, donde se promueve la participación interactiva con los contenidos de cada laboratorio; facilitándose la construcción del conocimiento, así como el almacenamiento, transmisión, recuperación, aplicación y enriquecimiento de los contenidos. Todo ello ocurre en forma autosuficiente, pues se brinda la posibilidad de un aprendizaje individual, al propio ritmo y adaptado a las necesidades de la vida cotidiana (Nájera \& Estrada, 2007: 94).

Como resultado de la investigación descriptiva realizada por Nájera \& Estrada, se concluyó que los usuarios de los laboratorios virtuales consideran que las principales características que éstos deben tener son: variedad en los contenidos y actividades, sencillez en la redacción y el uso, dinamismo, alta calidad en todo tipo de contenido y cierto grado de "entretenimiento" e interactividad.

Por su parte, Mesa, Ramírez, Ceballos \& Quiles (2012) concluyen en su investigación que con el uso de laboratorios virtuales se desarrolla pensamiento crítico, y se fomenta el análisis, la creatividad y el desarrollo de la lógica. Por su parte, Herrera, Sepúlveda \& Santa Sofía D.I.E concluyen que los laboratorios virtuales favorecen el pensamiento lógico-matemático a partir del desarrollo de cuatro capacidades: la observación, la imaginación, la intuición y el razonamiento lógico. Al respecto, Arce (2004) afirma que

[...] el Laboratorio de Matemáticas establece una relación dialéctica entre materiales manipulativos y actividad matemática. La utilización de los materiales produce una actividad manipulativa en quienes los usan y, a su vez, se convierten en elementos generadores de actividad mental, dinámicas que se contraponen con la pasividad externa que manifiestan los estudiantes que escuchan la explicación de un profesor. (p. 1).

En conclusión, cualquier actividad educativa que utilice medios electrónicos en su proceso formativo podrá resultar exitosa siempre y cuando docentes e instituciones se esfuercen por garantizar que las herramientas por emplear respondan en términos de contenido, diseño instruccional y estén ubicados al mismo nivel cognitivo de sus receptores: los estudiantes.

\section{Descripción del proceso de diseño de la plataforma LMS}

La labor educativa consciente y deliberada es capaz de percibir los retos, uno de ellos la inclusión; no vista como el transporte de ciertos estratos a un sistema que los rechazó y alienó durante años, sino como la posibilidad de adaptar el sistema a los requerimientos específicos de cada nuevo estudiante.

El laboratorio virtual de matemáticas recoge todos los vacíos detectados en el rastreo de referencias (bibliográficas, cibergráficas, etc.), incluyendo aquellos mostrados recientemente por las pruebas PISA $^{6}$ y las experiencias particulares de los autores en su desempeño como docentes de Educación Superior. 
El material educativo multimedia está orientado a personas con un nivel de estudios de media básica (noveno grado) en adelante, docentes y en general, a cualquier público interesado en afianzar sus conocimientos sobre matemáticas. El contenido del laboratorio fue creado para un público abierto, aunque se fundamentó en las competencias académicas básicas requeridas por los estudiantes de Educación Superior. Finalmente, en el desarrollo del proyecto investigativo se realizó la caracterización del grupo focal (seis estudiantes de primer semestre de programas académicos diversos) con el fin de tener referentes para la elección de estrategias, métodos y técnicas que favorecieran la aprehensión de competencias matemáticas.

\section{Enfoque metodológico y bases pedagógicas}

La plataforma LMS se diseñó con un propósito educativo que se compone de algunos elementos como contenidos temáticos (guías conceptuales en material multimedia), actividades de refuerzo pedagógico (ejercicios y prácticas) $\mathrm{y}$ herramientas de interacción entre estudiante y tutor. Con ello se buscó promover el desarrollo de competencias matemáticas utilizando las TIC como instrumento para reforzar los conocimientos adquiridos en el aula de clase. La efectividad del proceso de enseñanza depende en gran medida de la planificación y organización del curso, por lo tanto, para el laboratorio definimos unas fases acordes al nivel de habilidades y competencias de los aprendices. Considerando que la teoría constructivista del aprendizaje se basa en la resolución de problemas y el pensamiento crítico, se desplegaron diversas propuestas que retaron al estudiante a medir sus conocimientos mediante actividades que tenían una metodología más lúdica que evaluativa.

La técnica didáctica elegida para dar cumplimiento a los propósitos perseguidos con el laboratorio fue la de aprendizaje basado en problemas, el cual puede definirse como un enfoque educativo orientado al aprendizaje y a la instrucción en el que los alumnos abordan problemas reales bajo la supervisión de un tutor (Colegio Tecnológico de Monterrey, 2000).
El ABP consiste en el planteamiento de una situación problema, donde su construcción, análisis y/o solución constituyen el foco central de la experiencia, y donde la enseñanza consiste en promover deliberadamente el desarrollo del proceso de indagación y resolución del problema en cuestión (Díaz-Barriga, 2006).

El uso del ABP como técnica didáctica requiere que los estudiantes y mentores modifiquen su conducta y sus actitudes, implica además que tomen conciencia de la necesidad de desarrollar una serie de habilidades para poder tener un buen desempeño en sus actividades de aprendizaje (Caiseda \& Dávila, 2006: 19). Finalmente, para la organización de la técnica didáctica $\mathrm{ABP}$ en el diseño del laboratorio virtual de matemáticas se tuvo en consideración un esquema que resume el proceso de diseño y desarrollo de los objetos virtuales de aprendizaje, tal como aparece a continuación:

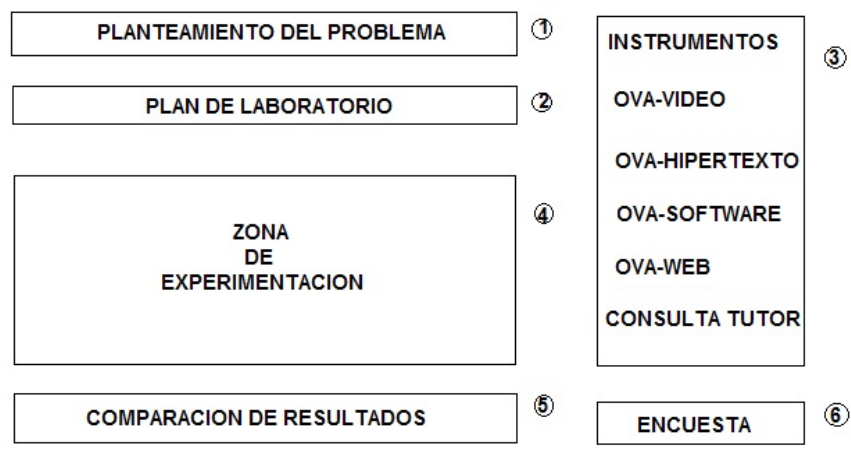

Figura 1. Esquema del laboratorio de matemáticas

1) Planteamiento del problema: consiste en la exposición de una situación real que requiere procesos lógicomatemáticos y operaciones para hallar soluciones.

2) Plan de laboratorio: opción de razonamiento o deducción de la solución, para detectar si existe correlación entre el éxito del proceso de pensamiento lógico matemático y el proceso meramente operativo y que básicamente consiste en la manera de proceder frente a la situación.

3) Instrumentos del laboratorio; serie de objetos virtuales de aprendizaje en multimedios con el fin de dotar de herramientas que se adapten a la forma de aprender 
Laboratorio virtual de matemáticas como estrategia didáctica para fomentar el pensamiento lógico

del estudiante: video, presentación, hipertexto, página y software libre.

4) Zona de experimentación: resultado del experimento, proceso operativo que requiere la mecánica y las técnicas procedimentales

5) Comparación de resultados: retroalimentación que resuma el proceso correcto para verificación de resultados.

6) Encuesta: que permita el sondeo de preferencias de medios y objetos.

\section{Resultados}

Adquisición de un hosting y del dominio www. escuelavirtual.co a través del cual no sólo se pone a disposición gratuita el laboratorio virtual de matemáticas sino otros cursos y proyectos en concordancia con nuestro compromiso de favorecer la democratización y pertinencia educativa. El portal web es diseñado en html5, de tal manera que se adapta a los diversos dispositivos móviles y computadores tradicionales, sin perder sus cualidades y posibilidades de accesibilidad a los cursos y otros enlaces. La administración de la página, dotada de moodle como campus virtual, es realizada por los autores del laboratorio y a la vez tutores de los demás cursos puestos a disposición de la sociedad.

Creación de comunidad académica a través del uso de redes sociales usando la imagen de escuela virtual y el posicionamiento hasta ahora logrado, así:

- Canal youtube: https://www.youtube.com/user/ LMSEscuelaVirtual

- Canal Facebook: https://www.facebook.com/ escuelavirtual.co

- Twitter: @escuelaLMS

- Diseño del laboratorio virtual en plataforma LMS, en el URL: http://www.escuelavirtual. co/index.php/cursos-disponibles/detail/3- matematicas/5-laboratorio-virtual-dematematicas

Para el laboratorio virtual de matemáticas se definieron las siguientes fases:

- Sensibilización (presentación): comprende toda la estrategia que permite el quebrantamiento del paradigma de la presencialidad, además de los bloqueos, taras y miedos provocados alrededor de las matemáticas. Incluye un examen clasificatorio que permitirá evidenciar la necesidad o no de desarrollar esta fase.

- Fundamentación (fase 1): se plantearon experimentos (situaciones problema) que poseen el requerimiento básico imprescindible para ingresar al mundo de las matemáticas; sistemas numéricos, ley de signos y propiedades de la potenciación.

- Matematización (fases 2 y 3): la capacidad de invertir los procesos mentales, realizar el recorrido de la realidad a la matemática permite un dominio práctico de los conceptos y la adquisición de conocimiento significativo y habilidades útiles, también consta de un examen clasificatorio que ayudará a determinar la estancia en esta fase o la continuación del proceso.

Ahora bien, el diseño y desarrollo de 213 OVA de temas matemáticos y 2.584 reproducciones en el canal de youtube ${ }^{7}$, enfocados aproximadamente el $80 \%$ al proceso operativo de las matemáticas y el $20 \%$ restante enfatizando en darle solución a problemas reales contribuyó a lograr un aprendizaje significativo y superior al matematizar situaciones cotidianas. Mientras el reconocimiento del laboratorio virtual de matemáticas por parte de la Coordinación de Bienestar Universitario de la Corporación Universitaria de Sabaneta como estrategia altamente valorada en del programa "cero deserción" escolar como consecuencia de la mortalidad académica presentada en algunas áreas del conocimiento, en este caso particular, el área de matemáticas la cual es transversal a todos los

7. Datos al 12 de enero de 2015. 
programas que se ofertan en la institución. Mientras la Corporación Universitaria de Sabaneta (UniSabaneta) posee un sistema de calificación de " 0 " a " 5 ", siendo " 0 " la calificación mínima y "5" la calificación máxima. Así, se realizó una comparación de los resultados obtenidos por los estudiantes del grupo focal para determinar regularidad o variaciones que permitieran obtener conclusiones interesantes. A continuación se muestra la tabla de los datos de las notas del primer y segundo examen:

\begin{tabular}{|l|c|c|}
\hline & $\begin{array}{c}\text { Calificación } \\
\text { examen 1 }\end{array}$ & $\begin{array}{c}\text { Calificación } \\
\text { examen 2 }\end{array}$ \\
\hline Estudiante 1 & 3.2 & 4 \\
\hline Estudiante 2 & 4.1 & 4 \\
\hline Estudiante 3 & 5 & 4.5 \\
\hline Estudiante 4 & 4 & 4.2 \\
\hline Estudiante 5 & 3.9 & 4.3 \\
\hline Estudiante 6 & 3.9 & 4.1 \\
\hline Media & 4,02 & 4.18 \\
\hline $\begin{array}{l}\text { Desviación } \\
\text { estándar }\end{array}$ & $\mathbf{0 . 5 8}$ & $\mathbf{0 . 1 9}$ \\
\hline $\begin{array}{l}\text { Coeficiente } \\
\text { de variación }\end{array}$ & $\mathbf{0 . 1 4}$ & $\mathbf{0 . 0 5}$ \\
\hline
\end{tabular}

Figura 2. Tabla de calificaciones, media, desviación y coeficiente de variación

Utilizando la fórmula para el cálculo de la media aritmética (ver figura 3) hallamos para cada juego de calificaciones de los seis estudiantes la media para el primer y segundo examen, notando que la diferencia entre ambas no fue significativa: sólo de un 3.2\%, lo que refleja un comportamiento regular con 2 meses de uso del laboratorio de matemáticas (LaMa); sin embargo, la diferencia entre las desviaciones típicas que fueron calculadas con la fórmula para una muestra (ver figura 4) presentó un comportamiento interesante, con una diferencia de 0.39 centésimas entre ambas desviaciones, reflejando que el grupo empezó a lograr una nivelación o equilibrio que se hizo más evidente en el cálculo del coeficiente de variación de Pearson que muestra qué tan parejo o semejante es el rendimiento del grupo de estudiantes, en donde se observó que para el segundo examen dicho coeficiente fue de tan sólo un $\mathrm{CV}=0.05$.

$$
\bar{X}=\frac{\sum_{i=1}^{k} X m_{i} \cdot f_{i}}{\sum_{i=1}^{k} f_{i}}
$$

Figura 3. Fórmula para el cálculo de la media aritmética de una muestra con datos agrupados ${ }^{8}$

$$
s=\sqrt{\frac{\sum_{i=1}^{N}(X i-\bar{X})^{2}}{N-1}}
$$

Figura 4. Fórmula para el cálculo de la desviación típica o estándar para una muestra

$$
C V=\frac{S}{|\bar{x}|}
$$

Figura 5. Fórmula para el cálculo del coeficiente de variación de Pearson ${ }^{9}$

Así, se hace necesario mostrar de manera directa el efecto del laboratorio virtual de matemáticas (LaMa) sobre la calificación teniendo en cuenta los progresos y retrocesos cuantitativos de cada estudiante. Para lograr determinar tal influencia, calculamos el coeficiente de correlación lineal de Pearson, tomando como variables por relacionar las horas que la plataforma LMS Moodle arroja como información de conexión para cada estudiante y como segunda variable el promedio de calificaciones final, tal como se muestra en la siguiente tabla: 
Laboratorio virtual de matemáticas como estrategia didáctica para fomentar el pensamiento lógico

\begin{tabular}{|l|c|c|}
\hline & Horas LaMa & Nota final \\
\hline Estudiante 1 & 7.2 & 3.9 \\
\hline Estudiante 2 & 4.3 & 3.9 \\
\hline Estudiante 3 & 12.7 & 4.3 \\
\hline Estudiante 4 & 8.1 & 4 \\
\hline Estudiante 5 & 16.8 & 4.5 \\
\hline Estudiante 6 & 22 & 4.3 \\
\hline
\end{tabular}

Figura 6. Tabla de relación entre las horas de conexión del estudiante y su progreso académico

$-1 \leq r=\frac{\operatorname{Cov}(X, Y)}{s_{X} s_{Y}}=\frac{\sum_{t=1}^{n}\left(X_{t}-\bar{X}\right) *\left(Y_{t}-\bar{Y}\right)}{\left.\sqrt{\sum_{t=1}^{n}\left(X_{t}-\bar{X}\right)^{2}} * \sqrt{\sum_{t=1}^{n}\left(Y_{t}\right.}-\bar{Y}\right)^{2}} \leq+1$

Figura7. Fórmula para el cálculo del coeficiente de correlación lineal de Pearson ${ }^{10}$

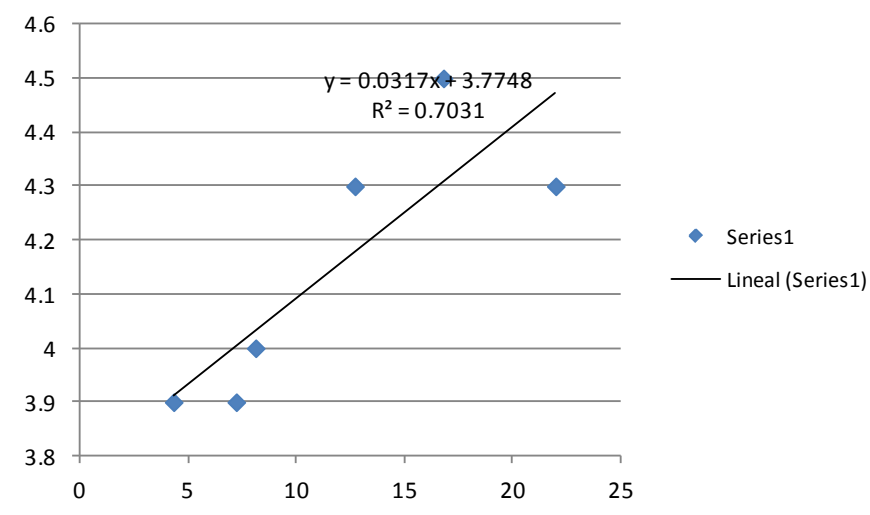

Figura 8. Coeficiente de correlación lineal y recta de regresión

Utilizando para el cálculo del coeficiente de correlación lineal la fórmula de Pearson (ver figura 7), donde cabe anotar que el coeficiente, por ser dos variables directamente proporcionales, oscilará entre " 0 " y " 1 ", siendo cero el mínimo coeficiente, lo que revela que no existe ninguna correlación entre las variables y "uno" el máximo coeficiente que representa una correlación total. El coeficiente de correlación de 0.7031 es considerable, estableciendo una relación fuerte entre el número de horas que un estudiante dedica al trabajo independiente en el laboratorio de matemáticas y los resultados de sus calificaciones.

Consultados sobre el medio preferido para el logro del aprendizaje respondieron de la siguiente manera:

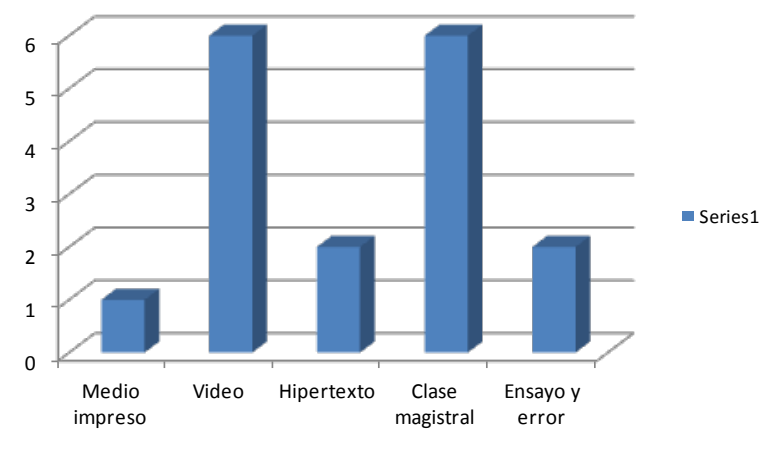

Figura 9. Medio preferido de aprendizaje

Los resultados eran predecibles considerando que es una generación que se desarrolló bajo un modelo de educación presencial, por tanto se favorece la elección de la clase magistral y el video, que emula de gran manera la metodología agregándole como valor adicional el hecho de poder ser reproducido en dispositivos móviles, asegurando la portabilidad del aula, de las lecciones a cualquier lugar.

\section{Conclusiones}

La inclusión educativa debe ir más allá de la inserción de estudiantes a instituciones que por motivos económicos u horarios, no habían podido ingresar.

Es necesario el estudio de las nuevas condiciones para evitar la deserción temprana y un nuevo rechazo por parte del sistema.

\section{Ibidem.}


El impacto de la tecnología y la concerniente digitalización que vivimos en la actualidad, necesariamente innova el proceso educativo para docentes y discentes, abre nuevas oportunidades y métodos para enseñar y aprender; exige una planeación diferente por parte del docente y exige del estudiante desempeñar no sólo su rol como alumno, sino aprovechar la interactividad para crear una relación social con su entorno.

Para operacionalizar la educación a distancia es conveniente tener presentes las siguientes premisas:

- la interactividad, como actividad fundamental del proceso de aprendizaje;

- la interconectividad entre temas, conocimientos y experiencias;

- la exploración de nuevas posibilidades de aprendizaje y desarrollo;

- el vínculo aprendizaje-materiales- orientadorestudiante;

- el acceso a diferentes escenarios de aprendizaje;

- y la combinación de opciones multimediales de logro de experiencias de aprendizaje.

El manejo de herramientas tecnológicas entonces enriquece y acerca el contenido de los objetos virtuales de aprendizaje, se hace muy necesario el uso de tabletas digitalizadoras, editores de video y sonido, cámaras de video, y obviamente el dominio administrativo de plataformas LMS para brindar una experiencia completa y versátil de enseñanza-aprendizaje.

El laboratorio virtual de matemáticas es una herramienta funcional, con resultados concretos que permiten considerarla en el tiempo como un apoyo permanente a las clases presenciales para facilitar al estudiante con dificultades de aprendizaje encontrar un nivel homogéneo con el grupo.
Es necesario así en todas las instituciones de Educación Superior, pero preponderante y urgentemente en aquellas cuyo nicho son los estudiantes trabajadores con horarios dispuestos para ello, la capacitación a los docentes en TIC para la realización de objetos virtuales de aprendizaje que llenen las expectativas del estudiante y se conviertan en un real apoyo de la enseñanza de las matemáticas.

La educación virtual puede lograr vencer la resistencia del estudiante dejando a un lado la pretensión de ser sustitutiva y convirtiéndola en un apoyo, así poco a poco ganará su lugar sin rupturas ni prevenciones.

El estudiante con ceses académicos prolongados trae la sensación de que ha olvidado todo y al enfrentarse a grupos numerosos heterogéneos en donde se encuentran jóvenes desde los 17 años recién salidos de la educación media con apropiadas habilidades y conocimientos matemáticos, sufre entonces de angustia y bloqueos aún mayores que pueden ser aliviados con la ayuda de la virtualidad para que el aprendizaje pueda ser regulado por este mismo, al poder detener, repetir y hasta solicitar material de estudio al docente.

El laboratorio virtual de matemática le permite al estudiante-trabajador poder acceder a las clases, a materiales y ayudas desde dispositivos móviles hasta computadores de escritorio permitiendo aprovechar tiempos muertos en sus jornadas laborales o en sus desplazamientos.

Asimismo, le posibilita al estudiante una comunicación directa y personalizada al estudiante que públicamente no expresa sus necesidades o inquietudes con respecto al tema tratado de manera presencial.

Una plataforma LMS permite impulsar en las instituciones de Educación Superior la asesoría virtual en todas las disciplinas. Para la generación que transita de la educación presencial a educación virtual, es mucho más fácil asimilar los contenidos a través del video educativo. 
Laboratorio virtual de matemáticas como estrategia didáctica para fomentar el pensamiento lógico

\section{Referencias}

Arce, J. (2004). El Laboratorio de Matemáticas. Área de Educación Matemática. Instituto de Educación y Pedagogía, Universidad del Valle. Documento Interno de Trabajo.

Bastán, M., \& Elguero, C. (2005). El escenario sociocultural en la formación matemática del sujeto adulto. Una indagación en alumnos del Nivel Medio. Premisa (Revista de la Sociedad Argentina de Educación Matemática), 7(27), 2335

Caiseda, C., \& Dávila, E. (2006). El aprendizaje basado en problemas y proyectos: una estrategia de integración. Universidad Interamericana de Puerto Rico.

Cangiani, S., \& Zabert, A. (2008). Uso de Multimedios para la Enseñanza de la Matemática.

Claro, M. (2010). Impacto de las TIC en los aprendizajes de los estudiantes. Estado del arte. Documentos de Proyectos.

Colegio Tecnológico de Monterrey (2000). Las técnicas didácticas en el modelo educativo del Tecnológico de Monterrey.
Chavarría, J., \& Carvajal, C. A. (2008). Producción de multimedia: una experiencia en el campo de las matemáticas. Cuadernos de Investigación y Formación en Educación Matemática, (4).

De Chaves, A. A., \& Peña, L. M. M. (2006). Reflexión sobre los procesos metodológicos de enseñanza y su incidencia en la cognición del estudiante de Educación Superior. Revista Ciencias de la Salud, 4.

Díaz Barriga Arceo, F. (2006). Enseñanza situada: vínculo entre la escuela y la vida. Editorial McGraw-Hill. México.

García, M. L., \& Benítez, A. A. (2011). Competencias Matemáticas Desarrolladas en Ambientes Virtuales de Aprendizaje: el Caso de MOODLE. (Spanish). Formación Universitaria, 4(3), 31-41. Doi:10.4067/S0718-50062011000300005

Granados, L. F. M., Urueña, L. A. L., \& Gamba, M. M. (2009). Análisis de interacciones en foro y chat: consolidación de grupo y liderazgo comunicativo en un curso de lógica matemática1.RIED. Revista Iberoamericana de Educación a Distancia, 12(2), pp. 189-210. 